\title{
First principles results of structural and electronic properties of $\mathrm{ZnS}$ clusters
}

\author{
D L LALSARE and ANJALI KSHIRSAGAR* \\ Department of Physics, University of Pune, Pune 411 007, India
}

MS received 18 February 2012

\begin{abstract}
We present results of the study of $\mathrm{Zn}_{n} \mathrm{~S}_{n}(1 \leq n \leq 9)$ clusters, using the density functional formalism and projector augmented wave method within the generalized gradient approximation. Along with the structural and electronic properties, nature of bonding and overall stability of clusters has been studied.
\end{abstract}

Keywords. Projector augmented wave method; fragmentation paths; HOMO-LUMO gap.

\section{Introduction}

In recent years group II-VI and III-V semiconductor nanostructures have been extensively studied. A modification of the electronic properties of these semiconductor materials with reduction in size is a field of growing interest. Nanosized clusters of these semiconductors have high optical transition probabilities for absorption and luminescence. Use of these nanosized structures in wide range of optical applications such as optical sensitizers (Sebastian and Ocampo 1996), solar energy converters (Ndukwe 1996), photoresistors, X-ray detectors etc has been demonstrated. Due to high surface to volume ratio in the nanosize regime, the nanoclusters are also used as chemical catalysts (Niemegeers and Burgelman 1997).

Luminescence of group II-VI semiconductors is still an extremely active area of research. Semiconductor $\mathrm{ZnS}$ crystals doped with transition metal or rare earth elements have been used as phosphors. $\mathrm{ZnS}$ is known to be a direct bandgap semiconductor having a wide bandgap of $3.68 \mathrm{eV}$. The HOMO-LUMO gap for $\mathrm{ZnS}$ nanoclusters can be tuned over a wide range by changing the cluster size or by doping with impurity atoms. These facts have motivated many researchers to synthesize doped or undoped quantum dots of $\mathrm{ZnS}$ and to simulate and study clusters and surfaces of $\mathrm{ZnS}$. Theoretical studies on small stoichiometric $\mathrm{Zn}_{n} \mathrm{~S}_{n}$ clusters $(1 \leq n \leq 9)$, using density functional theory (DFT) at the B3LYP (Becke 1993; Lee et al 1988) level, have concluded that tetramer $\left(\mathrm{Zn}_{4} \mathrm{~S}_{4}\right)$ is the last planar minimum energy cluster and for larger three-dimensional clusters the pertinent building blocks are $\mathrm{Zn}_{2} \mathrm{~S}_{2}$ four-membered rings and $\mathrm{Zn}_{3} \mathrm{~S}_{3}$ six-membered rings (Burnin et al 2005). Small stoichiometric $\mathrm{Zn}_{i} \mathrm{~S}_{i}$ clusters $(1 \leq i \leq 9)$ and very small

\footnotetext{
*Author for correspondence (anjali@ physics.unipune.ac.in)
}

non-stoichiometric $\mathrm{ZnS}$ clusters have also been investigated using DFT (Katircioğlu and Erkoc 2001; Maxtain et al 2001; Chuchev and BelBruno 2005; Hamad and Catlow 2005). Synthesis of $\mathrm{ZnS}$ quantum dots, with narrow size distribution using wet chemical method, predicted presence of cubic as well as hexagonal phase (Kumbhojkar et al 2000). Effective mass approximation, with infinite and finite potential and empirical pseudopotential method were used to understand the behaviour theoretically. A novel synthesis method reported tetrahedral structures for zinc surrounded by four sulfur atoms (Verma et al 1995). Recent mass spectrometric experiments have indicated the possibility of some homo-nuclear bonding within larger clusters (Chuchev and BelBruno 2005). Inorganic chemists have been exploring metal-sulfide bonds for quite a long time.

In our present work, we have carried out density functional study of stoichiometric $Z_{n} \mathrm{~S}_{n}(n=1$ to 9) clusters. We also probe the presence of $\mathrm{Zn}-\mathrm{Zn}$ and $\mathrm{S}-\mathrm{S}$ interactions in these clusters. The single particle energy levels can be used to explain photoluminescence spectra of small to medium nanoparticles. Information regarding the binding in these clusters is an aid to analyse materials for device applications.

\section{Methodology and computational details}

We have carried out all calculations using first principles pseudopotential method based on Kohn-Sham density functional theory (DFT) (Hohenberg and Kohn 1964; Kohn and Sham 1965) as implemented in Vienna ab initio simulation package (VASP) (Kresse and Furthmüller 1996a,b, 2001). We used plane wave basis set with a maximum plane wave cut-off energy of $280 \cdot 0 \mathrm{eV}$. The interaction between ions and electrons is described by pseudopotential within the framework of generalized gradient approximation (GGA) based on projector augmented wave (PAW) method (Blöchl 1994; Kresse and Joubert 1999). The exchange-correlation energy 
functional is represented by Perdew-Burke-Ernzerhoff (PBE) parametrization (Perdew et al 1996). The valence electron configurations used for $\mathrm{Zn}$ and $\mathrm{S}$ are $3 d^{10} 4 s^{2}$ and $3 s^{2} 3 p^{4}$, respectively.

For structural optimization, we have used a supercell of dimension $20 \times 20 \times 20 \AA^{3}$ with periodic boundary conditions. We have carried out calculations for cell sizes up to $30 \times 30 \times 30 \AA^{3}$ and have found that the total energy changed only marginally beyond $20 \AA^{3}$ whereas the computational cost increased with size as the number of plane waves used increased with size. The largest cluster studied in this work, $\mathrm{Zn}_{9} \mathrm{~S}_{9}$, is around $6.8 \AA$ in size. Even when we use the supercell of $20 \times 20 \times 20 \AA^{3}$, we thus have a vacuum region of 6-7 $\AA$. The electron wave-functions vanish well within the cell. The separation between the cluster and its images is roughly $14 \AA$. Therefore, the possibility of any long range interaction is negligibly small. The Brillouin zone is sampled by a single k-point ( $\Gamma$ point). The convergence criteria used are $0.0001 \mathrm{eV}$ for energy and $0.001 \mathrm{eV} / \AA$ for forces on ions.

For each system, we have spanned many possible geometries with and without symmetry considerations and by interchanging zinc and sulfur atoms to ensure that the possible lowest energy geometry has been found. Hereafter the lowest energy geometry is referred to as the global minimum (GM) and the immediate next higher energy geometry is referred to as the first local minimum (FLM).

\section{Results and discussion}

We have compared our results with earlier results (Maxtain et al 2001; Burnin et al 2005; Chuchev and BelBruno 2005). The overall agreement is satisfactory. In this work we have studied some additional features like nature of bonding, possibility of homo-nuclear bonding and overall stability of clusters. To determine stability of clusters, we have calculated the binding energy per monomer as defined below

$$
E_{\mathrm{b}}=\left[n E(\mathrm{Zn})+n E(\mathrm{~S})-E\left(\mathrm{Zn}_{n} \mathrm{~S}_{n}\right)\right] / n .
$$

To determine stability of the clusters with respect to addition or removal of an electron, we have calculated the vertical detachment energy (VDE) and electron affinity (EA) defined as follows

$$
\begin{aligned}
& \operatorname{VDE}\left[\mathrm{Zn}_{n} \mathrm{~S}_{n}\right]=E\left(\mathrm{Zn}_{n} \mathrm{~S}_{n}^{+}\right)-E\left(\mathrm{Zn}_{n} \mathrm{~S}_{n}\right), \\
& E A\left[\mathrm{Zn}_{n} \mathrm{~S}_{n}\right]=E\left(\mathrm{Zn}_{n} \mathrm{~S}_{n}\right)-E\left(\mathrm{Zn}_{n} \mathrm{~S}_{n}^{-}\right) .
\end{aligned}
$$

To determine the most favourable channel for fragmentation of cage-like structure into smaller building blocks like $\mathrm{Zn}_{1} \mathrm{~S}_{1}$, $\mathrm{Zn}_{2} \mathrm{~S}_{2}, \mathrm{Zn}_{3} \mathrm{~S}_{3}$, etc, we have calculated the fragmentation energy as

$$
\mathrm{FE}\left[\mathrm{Zn}_{n} \mathrm{~S}_{n}\right]=\sum_{i} k_{i} E\left(\mathrm{Zn}_{i} \mathrm{~S}_{i}\right)-E\left(\mathrm{Zn}_{n} \mathrm{~S}_{n}\right)
$$

where $k_{i}$ is the number of $\mathrm{Zn}_{i} \mathrm{~S}_{i}$ units in which the cluster $\mathrm{Zn}_{n} \mathrm{~S}_{n}$ fragments such that $\sum_{i} k_{i} \cdot i=n$.
To discuss the stability of a cluster relative to its neighbours, we have calculated the second order difference in total energy as follows

$$
\begin{aligned}
\Delta^{2} E\left(\mathrm{Zn}_{n} \mathrm{~S}_{n}\right)= & E\left(\mathrm{Zn}_{n-1} \mathrm{~S}_{n-1}\right)+E\left(\mathrm{Zn}_{n+1} \mathrm{~S}_{n+1}\right) \\
& -2 E\left(\mathrm{Zn}_{n} \mathrm{~S}_{n}\right)
\end{aligned}
$$

To describe the nature of bonding, delocalization of charge and other related properties, we have plotted isosurfaces of types (i) total charge density plots to understand overall nature of bonding in the cluster, (ii) difference charge density plots which represent the difference between total charge density of the cluster and superpositioned atomic change densities of the constituent atoms. This difference charge density explicitly shows change in the electronic charge density on formation of a cluster from its constituent atoms, (iii) partial charge density plots to bring out the contribution of each molecular orbital (MO) to the total charge density. It can be used to discuss the nature of individual molecular orbital, type of hybridization, overlap of molecular orbitals and delocalization of charge in the cluster and (iv) electron localization function (ELF) plots to estimate the localization of charge in the cluster. A value of 1 for ELF represents $100 \%$ localization of the electrons whereas a value of 0.5 indicates electron gas-like behaviour.

Our results for the structural properties of global and local minimum structures match to a good extent with previous reports (Maxtain et al 2001; Burnin and BelBruno 2002; Burnin et al 2005; Hamad and Catlow 2005). However, properties like binding energy and HOMO-LUMO gap show deviations from earlier results. This may be due to different methods of calculation, different parametrization used for the exchange-correlation energy functional and different basis sets used for simulation. In the following, we report our results.

\section{$3.1 \mathrm{Zn}_{1} \mathrm{~S}_{1}$}

Being a monomer, it has only one possible geometry, which is linear as shown in figure 1. Bond length and binding energy are smaller compared to the bulk values and are $2.056 \AA$ and $2.65 \mathrm{eV}$, respectively. Reported bond lengths in earlier published works vary from $2.05 \AA$ to $2 \cdot 13 \AA$. The total charge density plot as shown in figure 2(a) indicates that the bond between zinc and sulfur atoms in the monomer is partly covalent due to overlap of $s p$ hybridized orbital of zinc atom and $p$ orbital of sulfur atom, but has more ionic character due to transfer of $4 s$ electrons of zinc atom to partially filled $3 p$ orbitals of sulfur atom as shown in difference charge density plot of figure 3(a). A positive value around the $\mathrm{S}$ atom indicates that the electronic charge density in the cluster is more than the superpositioned atomic charge density while a negative value around the $\mathrm{Zn}$ atom indicates that charge density has decreased on formation of dimer. Thus charge is given from $\mathrm{Zn}$ atom to $\mathrm{S}$ atom in $\mathrm{ZnS}$ monomer. 


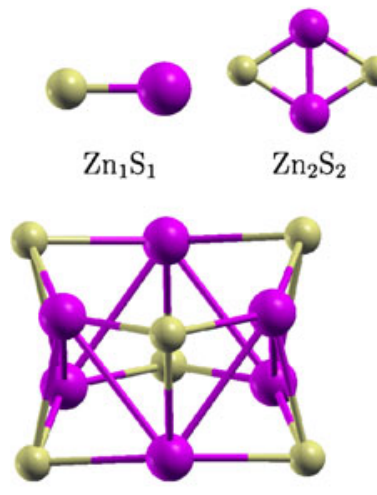

$\mathrm{Zn}_{6} \mathrm{~S}_{6}$
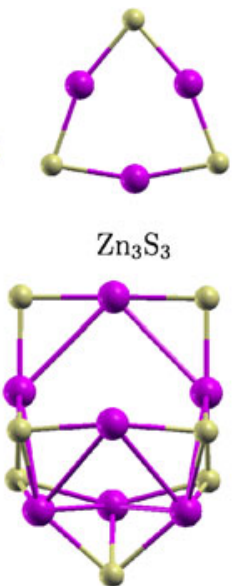

$\mathrm{Zn}_{7} \mathrm{~S}_{7}$

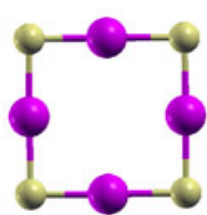

$\mathrm{Zn}_{4} \mathrm{~S}_{4}$

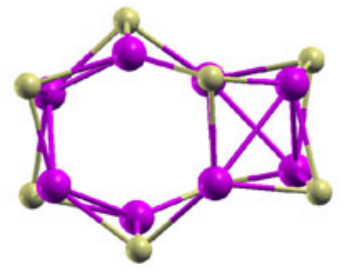

$\mathrm{Zn}_{8} \mathrm{~S}_{8}$

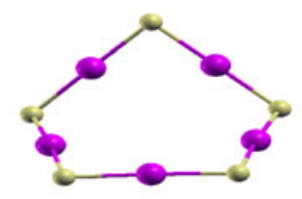

$\mathrm{Zn}_{5} \mathrm{~S}_{5}$

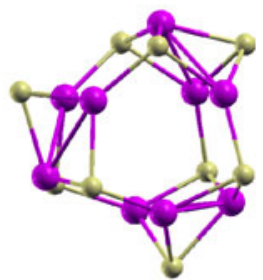

$\mathrm{Zn}_{9} \mathrm{~S}_{9}$

Figure 1. Lowest energy structures of $\mathrm{Zn}_{n} \mathrm{~S}_{n}$ with $n=1-9$. In this and following figures, dark, large (purple) spheres are zinc atoms and light, small (yellow) spheres are sulfur atoms.

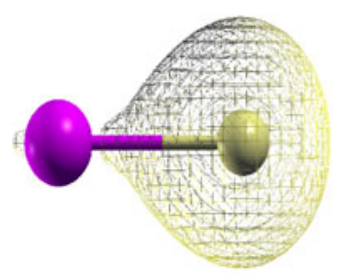

(a) $\mathrm{Zn}_{1} \mathrm{~S}_{1}$

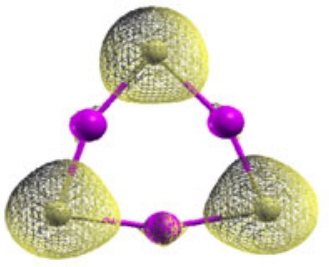

(b) $\mathrm{Zn}_{3} \mathrm{~S}_{3}$

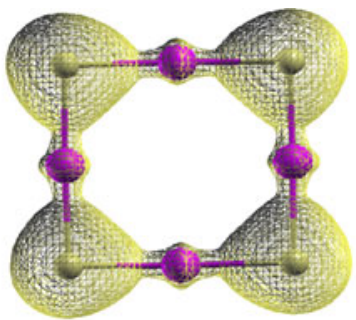

(c) $\mathrm{Zn}_{4} \mathrm{~S}_{4}$

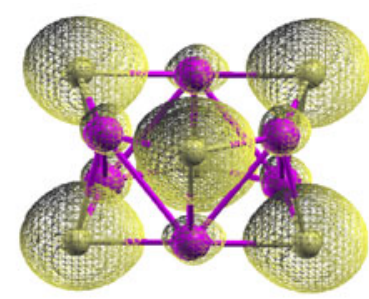

(d) $\mathrm{Zn}_{6} \mathrm{~S}_{6}$

Figure 2. Total charge density plots drawn at $1 / 3 \mathrm{rd}$ of maximum iso value.

\section{$3.2 \mathrm{Zn}_{2} \mathrm{~S}_{2}$}

Both the global and local minima for this cluster are planar ring-like structures. The global structure attains a rhombuslike geometry and favours ionic bonding due to alternately placed zinc and sulfur atoms in the cluster. Thus the cluster has higher binding energy of $4.66 \mathrm{eV}$ per $\mathrm{ZnS}$ monomer. This is due to the additional homo-nuclear S-S bonding. Partial charge density plots in figure 4(a) show homo-nuclear interaction between two sulfur atoms due to overlap of $p$ orbitals. $\mathrm{Zn}-\mathrm{S}$ bond length is more than that in monomer by $0 \cdot 19 \AA$. This is due to strong repulsion between lone pair of electrons of sulfur atoms. FLM is a near-square structure and is $0.94 \mathrm{eV}$ higher in energy than global minimum because it neither has an ionic bond nor has any homo-nuclear interaction across the cluster.

\section{$3.3 \mathrm{Zn}_{3} \mathrm{~S}_{3}$}

Global minimum is like an equilateral triangle with sulfur atoms on three vertices and zinc atoms at the mid points of three sides as shown in figure 1 . All $\mathrm{Zn}-\mathrm{S}$ bond lengths are same and are equal to $2 \cdot 17 \AA$ resulting in each side of the triangle to be $4.34 \AA$. All $\mathrm{S}-\mathrm{Zn}-\mathrm{S}$ bond angles are equal to $160^{\circ}$ and the $\mathrm{Zn}-\mathrm{S}-\mathrm{Zn}$ angles are $80 \cdot 2^{\circ}$. Symmetry of the cluster is $D_{3 h}$. The difference density plot shown in figure 3(b) indicates that the sulfur atoms have gained charge from the zinc atoms. Thus bonds between zinc and sulfur atoms are partly ionic. There is also build-up of charge in the central region of the cluster as compared to superposition of atomic charge density. There are six hetero-nuclear bonds between $\mathrm{Zn}$ and $\mathrm{S}$ atoms. The binding energy is $5.67 \mathrm{eV}$ per $\mathrm{ZnS}$ monomer. This increase is due to presence of weak homo-nuclear interaction between sulfur atoms as evident from partial charge density plot of figure 4(b). The difference between the vertical detachment energy and the electron affinity is maximum for $\mathrm{Zn}_{3} \mathrm{~S}_{3}$. This indicates that it is the most stable cluster among all clusters reported in this work against addition or removal of an electron. Partial charge density plot of LUMO+1 orbital shows delocalization of charge at the centre of the cluster indicating its semi-metallic nature.

\section{$3.4 Z n_{4} S_{4}$}

The global minimum is a square ring-like structure. It is the most symmetric structure among all stoichiometric clusters 


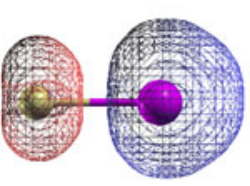

(a) $\mathrm{Zn}_{1} \mathrm{~S}_{1}$

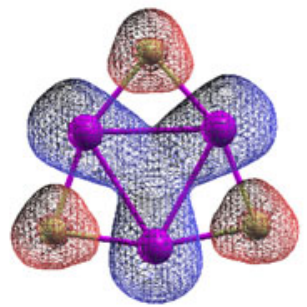

(b) $\mathrm{Zn}_{3} \mathrm{~S}_{3}$

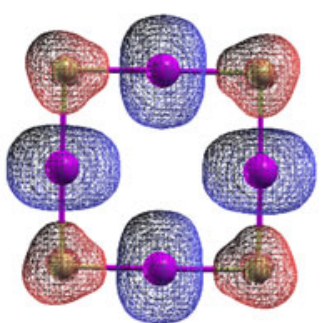

(c) $\mathrm{Zn}_{4} \mathrm{~S}_{4}$

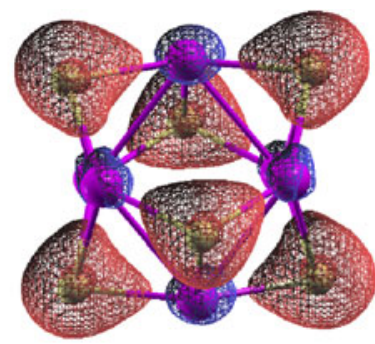

(d) $\mathrm{Zn}_{6} \mathrm{~S}_{6}$

Figure 3. Difference charge density (defined in text) plots drawn at $1 / 3$ rd of maximum iso value. Pink and blue colours of net, respectively represent positive and negative values for difference charge density. Charge density contributed by $3 d$ electrons of $\mathrm{Zn}$ atoms has been subtracted out for better view.

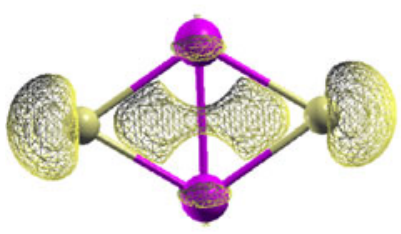

(a) $\mathrm{Zn}_{2} \mathrm{~S}_{2}-\mathrm{HOMO}-5$

Figure 4. Partial charge density for some typical clusters showing $\mathrm{S}-\mathrm{S}$ interaction. Plots are drawn for indicated molecular orbital at $1 / 4$ th of maximum iso value.

studied in this work and possesses $D_{4 h}$ point group symmetry as shown in figure 1 . All $\mathrm{Zn}-\mathrm{S}$ bond lengths are equal to $2 \cdot 15 \AA$ making each side of square $4.30 \AA$ and all $\mathrm{S}-\mathrm{Zn}-\mathrm{S}$ bond angles are almost linear. The $\mathrm{Zn}-\mathrm{S}-\mathrm{Zn}$ bond angles are $91^{\circ}$. FLM structure has $T_{d}$ symmetry and has distorted cubic structure with coordination number of three, but it is $1.11 \mathrm{eV}$ higher in energy than the global minimum. This is due to strained nonlinear $\mathrm{Zn}-\mathrm{S}$ bonds. In GM structure, there is no homo-nuclear interaction across the cluster and the coordination number is two. The binding energy is $5.87 \mathrm{eV}$ per $\mathrm{ZnS}$ monomer. HOMO-LUMO gap is $3 \mathrm{eV}$. It is interesting to note that the LUMO shows delocalization of charge at the centre of the cluster as shown in figure 5(a). It may be mentioned that the delocalization of MO is shifted down to $\mathrm{LUMO}$ whereas it is seen in $\mathrm{LUMO}+1$ or $\mathrm{LUMO}+2$ for other clusters. This is due to more pronounced overlap of $p$ orbitals of all sulfur atoms and $s p$ hybrid orbitals of all zinc atoms due to highly symmetric and planar geometry. It is the $\mathrm{Zn}-\mathrm{Zn}$ interaction that indicates the metallicity of a cluster. It is found to be largest in extent for $\mathrm{Zn}_{4} \mathrm{~S}_{4}$ cluster. Secondly, the energy difference between the HOMO and the lowest unoccupied and delocalized molecular orbital is smallest for $\mathrm{Zn}_{4} \mathrm{~S}_{4}$. This indicates that $\mathrm{Zn}_{4} \mathrm{~S}_{4}$ is more metallic than other clusters. However, the difference density plot also indicates presence of ionic character.

\section{$3.5 \mathrm{Zn}_{5} \mathrm{~S}_{5}$}

This is the first structure in the stoichiometric group which is not perfectly planar. As shown in figure 1, it is a pentagonal ring-like structure having $C_{1 h}$ point group symmetry, but the pentagon is formed by two planes with an angle of $93^{\circ}$ between them. The average $\mathrm{Zn}-\mathrm{S}$ bond length is $2.15 \AA$, the average $\mathrm{S}-\mathrm{Zn}-\mathrm{S}$ bond angle is $176^{\circ}$ and the average $\mathrm{Zn}-$ $\mathrm{S}-\mathrm{Zn}$ bond angle is $94.5^{\circ}$. The binding energy is $5.91 \mathrm{eV}$ per $\mathrm{ZnS}$ monomer. HOMO-LUMO gap is $3.32 \mathrm{eV}$ and is the highest among the entire group. Here also LUMO shows delocalization of charge at the centre of the cluster due to overlap of $p$ orbitals of all sulfur atoms and $s p$ hybrids of all zinc atoms. But the extent of delocalization is small compared to that in the $\mathrm{Zn}_{4} \mathrm{~S}_{4}$ cluster.

\section{$3.6 Z_{6} S_{6}$}

This is the first three dimensional cage-like structure in the stoichiometric group and has not been reported earlier. The global minimum is formed by two $\mathrm{Zn}_{3} \mathrm{~S}_{3}$ units bonded together by $\mathrm{Zn}_{2} \mathrm{~S}_{2}$ units as shown in figure 1. It has $C_{3 v}$ point group symmetry. GM is lower in energy than FLM by $0.43 \mathrm{eV}$ due to higher coordination number of three for all atoms. There is no homo-nuclear $\mathrm{Zn}-\mathrm{Zn}$ interaction across the cluster. Average $\mathrm{Zn}-\mathrm{S}$ bond length increases to $2.27 \AA$ compared to the planar structures and binding energy is $6.04 \mathrm{eV}$ per $\mathrm{ZnS}$ monomer. This increase in binding energy is because of higher coordination number for all the atoms due to formation of three-dimensional structure. HOMO-LUMO gap is $2.54 \mathrm{eV}$.

Delocalization of charge is shifted to higher orbital LUMO+1 as shown in figure 5(c). Ionicity of hetero-nuclear bonds decreases as seen from the difference charge density plot of figure 3(d). Instead of charge transfer, we find 


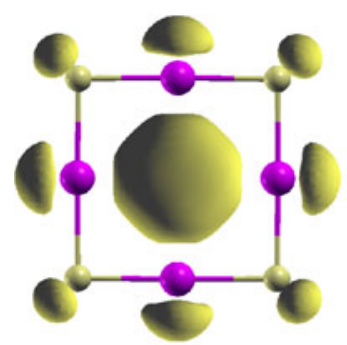

(a) $\mathrm{Zn}_{4} \mathrm{~S}_{4}$-LUMO

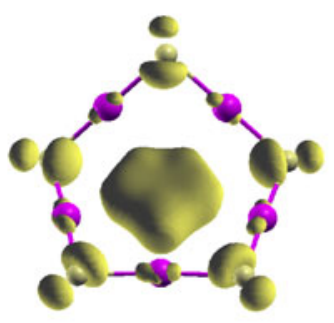

(b) $\mathrm{Zn}_{5} \mathrm{~S}_{5}$-LUMO

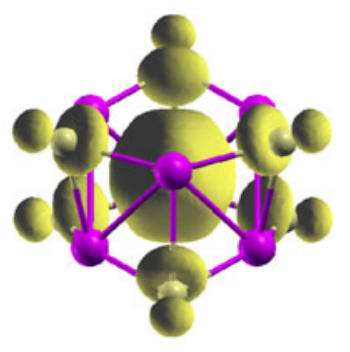

(c) $\mathrm{Zn}_{6} \mathrm{~S}_{6}-\mathrm{LUMO}+2$

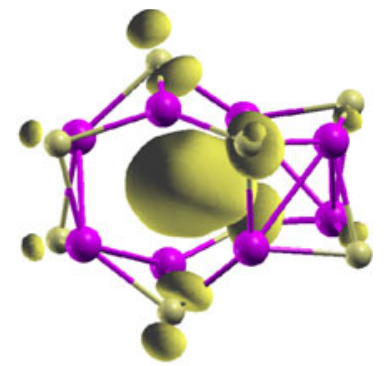

(d) $\mathrm{Zn}_{8} \mathrm{~S}_{8}-\mathrm{LUMO}+2$

Figure 5. Partial charge density plots drawn at $1 / 3 \mathrm{rd}$ of maximum iso value for some typical clusters showing delocalization of charge at centre of cluster. Plots are drawn for molecular orbital indicated in figure 6.

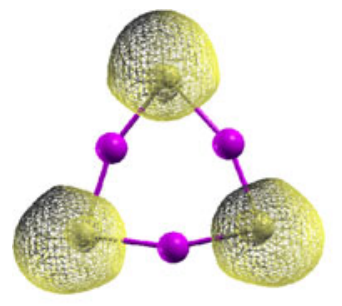

(a) $\mathrm{Zn}_{3} \mathrm{~S}_{3}$

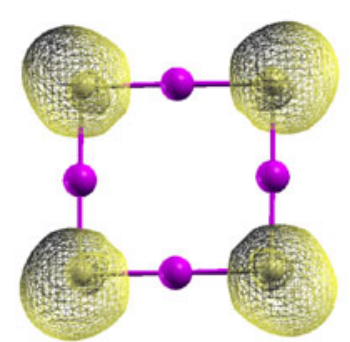

(b) $\mathrm{Zn}_{4} \mathrm{~S}_{4}$

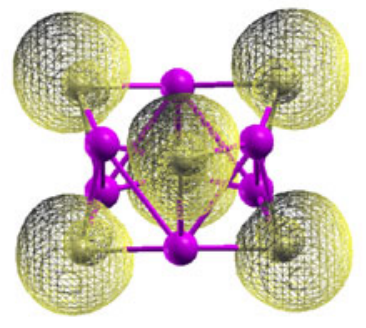

(c) $\mathrm{Zn}_{6} \mathrm{~S}_{6}$

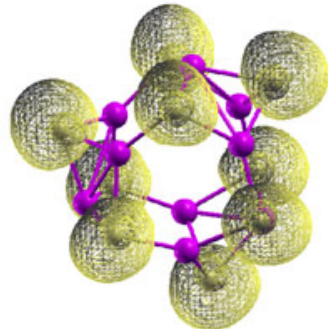

(d) $\mathrm{Zn}_{9} \mathrm{~S}_{9}$

Figure 6. Electron localization function plots drawn at a value of 0.65.

bonding orbitals resulting from $\mathrm{Zn} s$ and $\mathrm{S} p$ orbitals to be predominantly $\mathrm{S} p$-like and anti-bonding orbitals to have more $\mathrm{Zn} s$ character. Thus the three-dimensional geometry and higher coordination brings about a significant change in the nature of bonding. Local minimum is also a threedimensional structure with zinc atoms at the vertices and sulfur atoms above six faces of an octahedron. It is also formed from $\mathrm{Zn}_{3} \mathrm{~S}_{3}$ and $\mathrm{Zn}_{2} \mathrm{~S}_{2}$ units.

\section{$3.7 \mathrm{Zn}_{7} \mathrm{~S}_{7}$}

The global minimum is a basket-like structure formed by stacking one unit each of $\mathrm{Zn}_{3} \mathrm{~S}_{3}$ and $\mathrm{Zn}_{4} \mathrm{~S}_{4}$ as shown in figure 1. These two units are bonded together by $\mathrm{Zn}_{2} \mathrm{~S}_{2}$ units. This geometry is different from the one reported earlier (Maxtain et al 2001). Average $\mathrm{Zn}-\mathrm{S}$ bond length is $2.23 \AA$ and binding energy is $6.03 \mathrm{eV}$ per $\mathrm{ZnS}$ monomer. HOMOLUMO gap decreases to $2.13 \mathrm{eV}$. FLM is a planar ring-like structure which is higher in energy by $0.39 \mathrm{eV}$ than GM due to lower coordination number of two for all atoms and also due to less linear $\mathrm{S}-\mathrm{Zn}-\mathrm{S}$ bonds. There is no $\mathrm{Zn}-\mathrm{Zn}$ interaction across the cluster in GM. Delocalization of charge is seen at $\mathrm{LUMO}+1$.

\section{$3.8 \mathrm{Zn}_{8} \mathrm{~S}_{8}$}

As shown in figure 1, the global minimum for $\mathrm{Zn}_{8} \mathrm{~S}_{8}$ is a cage-like structure formed by $\mathrm{Zn}_{3} \mathrm{~S}_{3}$ and $\mathrm{Zn}_{2} \mathrm{~S}_{2}$ units having point group symmetry, $C_{2}$. Average $\mathrm{Zn}-\mathrm{S}$ bond length is $2.21 \AA$ and binding energy is $6.19 \mathrm{eV}$ per $\mathrm{ZnS}$ monomer. Delocalization of charge is shifted to higher orbital LUMO+1 as shown in figure 5(d). HOMO-LUMO gap is $2.75 \mathrm{eV}$. FLM is formed by stacking two units of $\mathrm{Zn}_{4} \mathrm{~S}_{4}$. It is higher in energy than the global minimum by $0 \cdot 87 \mathrm{eV}$.

\section{$3.9 Z n_{9} S_{9}$}

The global minimum is a cage-like structure formed by $\mathrm{Zn}_{3} \mathrm{~S}_{3}$ and $\mathrm{Zn}_{2} \mathrm{~S}_{2}$ units with an average $\mathrm{Zn}-\mathrm{S}$ bond length of $2.32 \AA$. The coordination number for all sulfur atoms is three but for zinc atoms it is four. The binding energy is $6.26 \mathrm{eV}$ per $\mathrm{ZnS}$ monomer. HOMO-LUMO gap is $2.81 \mathrm{eV}$. Delocalization of charge is shifted down to LUMO+1.

There are some common findings which we describe below. Homo-nuclear interaction between sulfur atoms is seen from partial charge density plots of $\mathrm{Zn}_{2} \mathrm{~S}_{2}$ and $\mathrm{Zn}_{3} \mathrm{~S}_{3}$ clusters as shown in figure 4. Delocalization of charge at centre of the ring-like structures occurs at LUMO of $\mathrm{Zn}_{4} \mathrm{~S}_{4}$ and $\mathrm{Zn}_{5} \mathrm{~S}_{5}$ clusters which are planar and semi-planar but it is shifted up to LUMO+2 for three-dimensional cagelike structures $\mathrm{Zn}_{6} \mathrm{~S}_{6}$ and $\mathrm{Zn}_{8} \mathrm{~S}_{8}$ as shown in figure 5 and to $\mathrm{LUMO}+1$ for $\mathrm{Zn}_{9} \mathrm{~S}_{9}$. This fact indicates that the planar structures are more metallic than cage-like structures. Isosurface plots drawn for electron localization function (ELF), as shown in figure 6 , indicate that there is no possibility of 


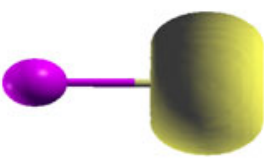

(a) $\mathrm{Zn}_{1} \mathrm{~S}_{1}-\mathrm{HOMO}$

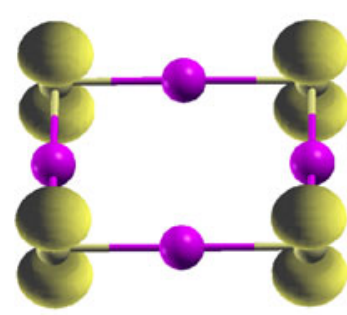

(e) $\mathrm{Zn}_{4} \mathrm{~S}_{4}$-HOMO

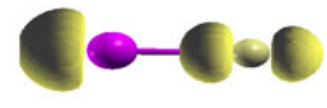

(b) $\mathrm{Zn}_{1} \mathrm{~S}_{1}$-LUMO

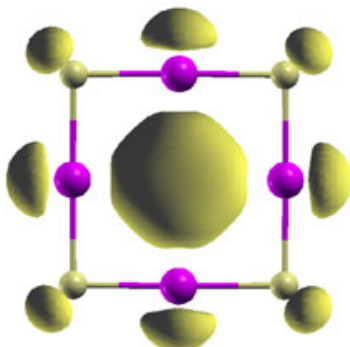

(f) $\mathrm{Zn}_{4} \mathrm{~S}_{4}$-LUMO



(c) $\mathrm{Zn}_{3} \mathrm{~S}_{3}-\mathrm{HOMO}$

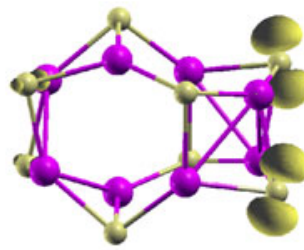

(g) $\mathrm{Zn}_{8} \mathrm{~S}_{8}-\mathrm{HOMO}$

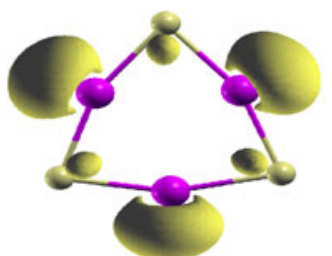

(d) $\mathrm{Zn}_{3} \mathrm{~S}_{3}$-LUMO

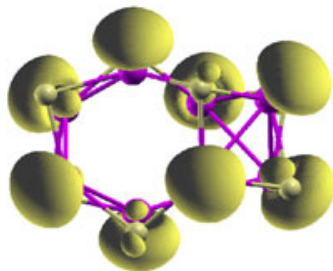

(h) $\mathrm{Zn}_{8} \mathrm{~S}_{8}$-LUMO

Figure 7. Partial charge density plots drawn at $1 / 3$ rd of maximum iso value.
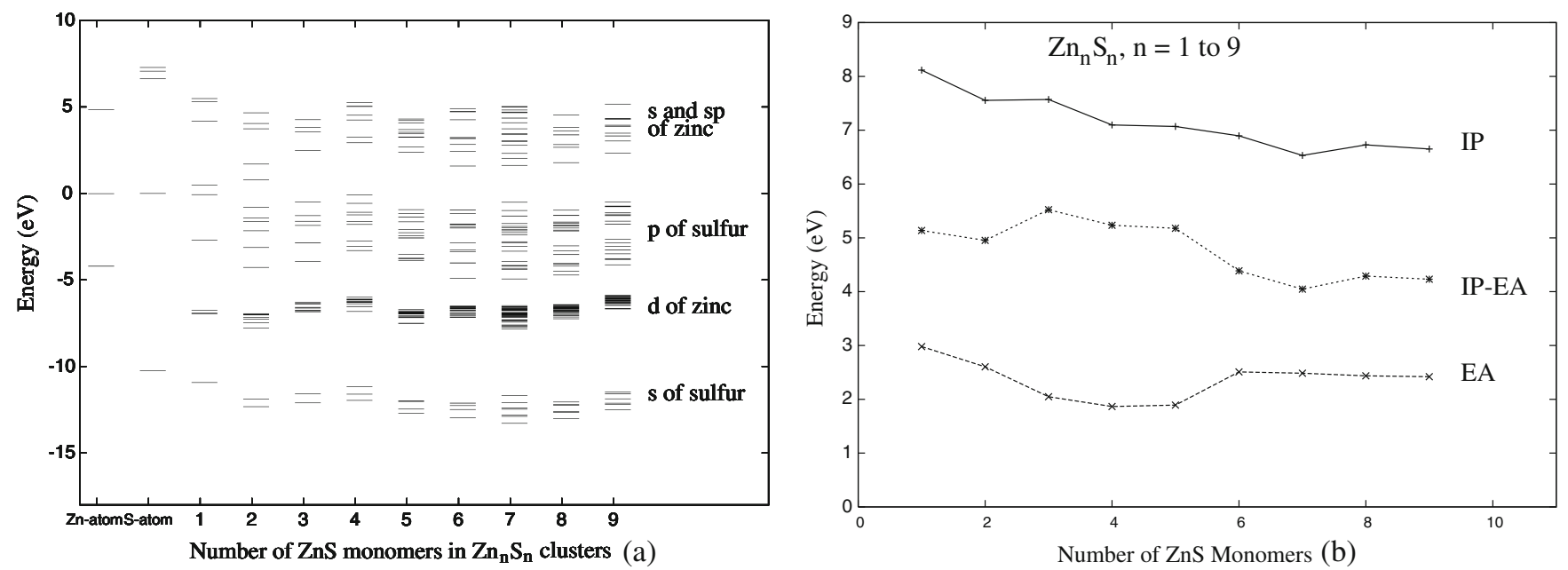

Figure 8. (a) Single particle energy level diagram for $\mathrm{Zn}_{n} \mathrm{~S}_{n}$ and (b) ionization potential and electron affinity vs number of $\mathrm{ZnS}$ monomers.

two zinc or sulfur atoms to be bonded to each other except very weak homo-nuclear interactions in some small clusters. Localization of charge around sulfur atoms is seen due to transfer of charge from zinc to sulfur atoms and hence bonding is partly ionic in nature. This type of bonding is also confirmed from plots of difference charge density. These plots, as shown in figure 3, depict that the entire charge is present on sulfur atoms for planar structures. This is due to electron transfer from the zinc atoms to sulfur atoms and hence strong polarization of sulfur atoms indicating mixed covalent and ionic bonding. Ionic character of $\mathrm{Zn}-\mathrm{S}$ bond is more dominating than the covalent character in all planar clusters as is evident from the isosurface plots drawn for total charge density for some typical global minimum structures in figure 2. However, the ionic character decreases and the covalent character increases gradually with increasing $n$. This is more evident at $n=5$ where structures start acquiring three-dimensional geometry. Isosurface plots of partial charge density, drawn for HOMO and LUMO of typical clusters in figure 7, indicate that except for $\mathrm{Zn}_{1} \mathrm{~S}_{1}$, HOMO is pure $p$ orbitals of sulfur and LUMO is a mixture of $p$ orbitals of sulfur and $s p$ hybrid orbitals of zinc atoms.

The HOMO-LUMO gap varies from 0.55 to $3.32 \mathrm{eV}$ as can be seen from the plots of single-particle energies in figure 8(a). It is maximum for $\mathrm{Zn}_{5} \mathrm{~S}_{5}$ cluster and minimum for $\mathrm{Zn}_{1} \mathrm{~S}_{1}$. The variation in HOMO-LUMO gap is mainly due to upward shift of both HOMO and LUMO in planar structures and shift of only HOMO in cage-like structures. Figure 8(b) shows that the difference between vertical detachment energy (VDE) and the electron affinity (EA), 

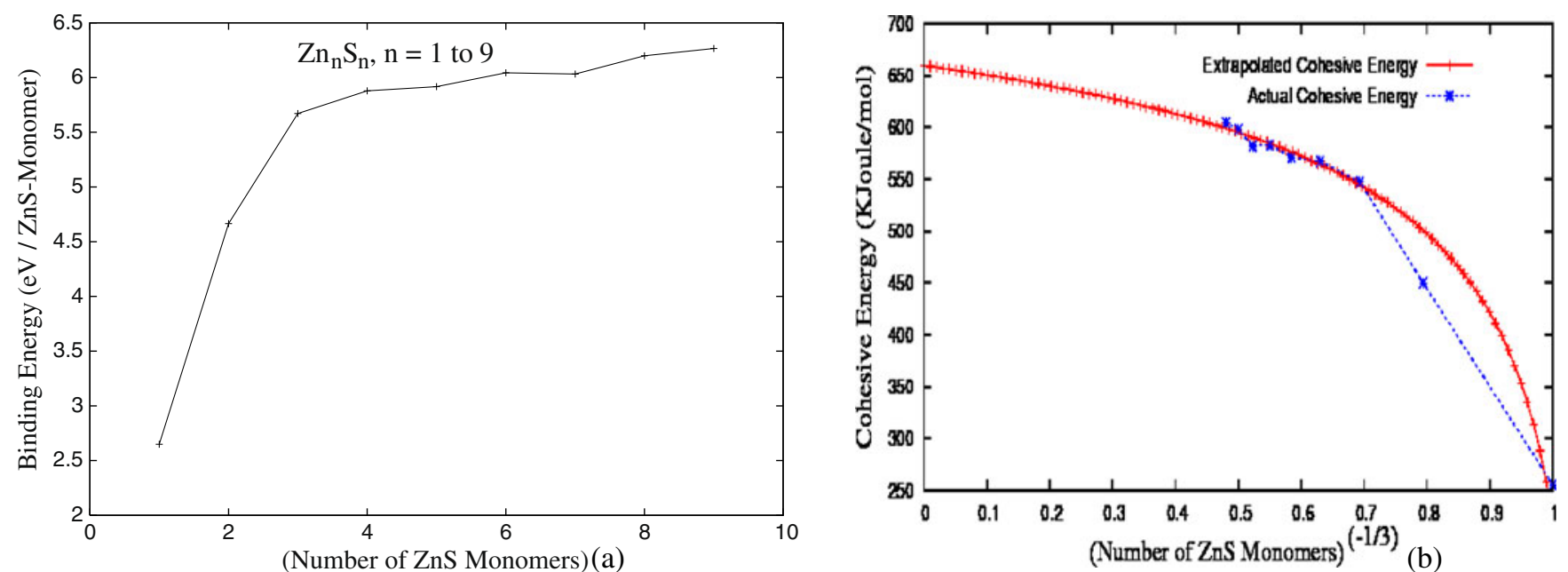

Figure 9. (a) Plot of binding energy vs number of $\mathrm{ZnS}$ monomers and (b) plot of binding energy per mole vs $1 / n^{1 / 3}$.

which is a measure of hardness, is maximum for $\mathrm{Zn}_{3} \mathrm{~S}_{3}$ cluster and is minimum for $\mathrm{Zn}_{7} \mathrm{~S}_{7}$ cluster. Hardness measures sensitivity of the electronegativity to change in the number of electrons. The graph shows that the clusters $\mathrm{Zn}_{n} \mathrm{~S}_{n}$ with $n=3$ to 5 , will resist a change in the number of electrons in comparison to other clusters and that $\mathrm{Zn}_{3} \mathrm{~S}_{3}$ is the most stable and $\mathrm{Zn}_{7} \mathrm{~S}_{7}$ is the least stable structure in the stoichiometric group with respect to addition or removal of an electron in the cluster.

As shown in graph of figure 9(a), the binding energy per $\mathrm{ZnS}$ monomer, calculated using (1) for all clusters, increases with size of the cluster. The plot of binding energy (in $\mathrm{kJ} / \mathrm{mol}$ ) vs inverse of cube-root of number of monomers in the cluster extrapolated to $n=\infty$ gives prediction for cohesive energy of bulk $\mathrm{ZnS}$ as shown in figure 9(b). The extrapolated value is equal to $660 \mathrm{~kJ} / \mathrm{mol}$ and is close to the reported theoretical $(563.36 \mathrm{~kJ} / \mathrm{mol}$ and $574 \pm 20 \mathrm{~kJ} / \mathrm{mol})$ and experimental $(607 \mathrm{~kJ} / \mathrm{mol})$ values (Maxtain et al 2001; Burnin et al 2005). The structural stability is also verified from the favourable paths for fragmentation of cagelike structures as shown in table 1 . We have calculated the fragmentation energies (FE) using (4) for all possible fragmentation channels.

Table 1 lists the most probable fragmentation channels $(\mathrm{FE}<10 \mathrm{eV})$ for larger clusters; the fragments are given in the second column. Value of FE indicates the energy needed to be supplied to fragment the cluster. A negative value for FE would mean that cluster would spontaneously fragment. All the values for the fragmentation energies are positive indicating that all the clusters studied are stable with respect to fragmentation into smaller $Z_{i} S_{i}$ units. The table shows that either $\mathrm{Zn}_{3} \mathrm{~S}_{3}$ or $\mathrm{Zn}_{4} \mathrm{~S}_{4}$ cluster is one of the constituent in the fragmentation process. $\mathrm{Zn}_{3} \mathrm{~S}_{3}$ is the most stable stoichiometric cluster and it serves as the building unit for larger cage-like structures. We have also studied non-stoichiometric clusters (results not presented here). Fragmentation analysis of all the clusters indicates that they prefer to fragment into
Table 1. Most probable fragmentation channels for $\mathrm{Zn}_{n} \mathrm{~S}_{n}$ with fragmentation energy $(\mathrm{FE})<10 \mathrm{eV}$.

\begin{tabular}{|c|c|c|c|c|c|c|}
\hline \multirow[b]{2}{*}{ System } & \multicolumn{5}{|c|}{ Number of units of fragments } & \multirow{2}{*}{$\begin{array}{c}\text { FE } \\
(\mathrm{eV})\end{array}$} \\
\hline & $\mathrm{Zn}_{1} \mathrm{~S}_{1}$ & $\mathrm{Zn}_{2} \mathrm{~S}_{2}$ & $\mathrm{Zn}_{3} \mathrm{~S}_{3}$ & $\mathrm{Zn}_{4} \mathrm{~S}_{4}$ & $\mathrm{Zn}_{5} \mathrm{~S}_{5}$ & \\
\hline \multirow[t]{4}{*}{$\mathrm{Zn}_{5} \mathrm{~S}_{5}$} & - & 1 & 1 & - & - & $2 \cdot 88$ \\
\hline & 1 & - & - & 1 & - & $3 \cdot 24$ \\
\hline & 2 & - & 1 & - & - & $6 \cdot 92$ \\
\hline & 1 & 2 & - & - & - & 7.74 \\
\hline \multirow{6}{*}{$\mathrm{Zn}_{6} \mathrm{~S}_{6}$} & - & - & 2 & - & - & $1 \cdot 88$ \\
\hline & - & 1 & - & 1 & - & $3 \cdot 06$ \\
\hline & 1 & - & - & - & 1 & $3 \cdot 85$ \\
\hline & 1 & 1 & 1 & - & - & $6 \cdot 73$ \\
\hline & 2 & - & - & 1 & - & $7 \cdot 09$ \\
\hline & - & 3 & - & - & - & $7 \cdot 55$ \\
\hline \multirow[t]{6}{*}{$\mathrm{Zn}_{7} \mathrm{~S}_{7}$} & - & - & 1 & 1 & - & 1.33 \\
\hline & 1 & - & 2 & - & - & $5 \cdot 01$ \\
\hline & - & 2 & 1 & - & - & $5 \cdot 83$ \\
\hline & 1 & 1 & - & 1 & - & $6 \cdot 19$ \\
\hline & 2 & - & - & - & 1 & 6.98 \\
\hline & 2 & 1 & 1 & - & - & $8 \cdot 86$ \\
\hline \multirow[t]{7}{*}{$\mathrm{Zn}_{8} \mathrm{~S}_{8}$} & - & - & - & 2 & - & $2 \cdot 20$ \\
\hline & - & - & 1 & - & 1 & 2.63 \\
\hline & - & 1 & 2 & - & - & $5 \cdot 51$ \\
\hline & 1 & - & 1 & 1 & - & $5 \cdot 88$ \\
\hline & - & 2 & - & 1 & - & $6 \cdot 70$ \\
\hline & 1 & 1 & - & - & 1 & 7.49 \\
\hline & 2 & - & 2 & - & - & 9.55 \\
\hline \multirow[t]{7}{*}{$\mathrm{Zn}_{9} \mathrm{~S}_{9}$} & - & - & - & 1 & 1 & 2.93 \\
\hline & - & - & 3 & - & - & 4.63 \\
\hline & - & 1 & 1 & 1 & - & $5 \cdot 81$ \\
\hline & 1 & - & - & 2 & - & $6 \cdot 17$ \\
\hline & 1 & - & 1 & - & 1 & $6 \cdot 60$ \\
\hline & - & 2 & - & - & 1 & 7.42 \\
\hline & 1 & 1 & 2 & - & - & $9 \cdot 48$ \\
\hline
\end{tabular}


Table 2. Second order difference in total energy $\Delta^{2} E$ for $\mathrm{Zn}_{n} \mathrm{~S}_{n}$ clusters.

\begin{tabular}{lr}
\hline System & $\Delta^{2} E(\mathrm{eV})$ \\
\hline $\mathrm{Zn}_{2} \mathrm{~S}_{2}$ & -0.82 \\
$\mathrm{Zn}_{3} \mathrm{~S}_{3}$ & 1.18 \\
$\mathrm{Zn}_{4} \mathrm{~S}_{4}$ & 0.43 \\
$\mathrm{Zn}_{5} \mathrm{~S}_{5}$ & -0.60 \\
$\mathrm{Zn}_{6} \mathrm{~S}_{6}$ & 0.72 \\
$\mathrm{Zn}_{7} \mathrm{~S}_{7}$ & -1.41 \\
$\mathrm{Zn}_{8} \mathrm{~S}_{8}$ & 0.57 \\
\hline
\end{tabular}

smaller stoichiometric clusters than non-stoichiometric clusters. Fragmentation channels with $\mathrm{Zn}, \mathrm{Zn}_{2}$ or $\mathrm{S}, \mathrm{S}_{2}$ are not favoured.

The second order difference in total energy, given by (5), indicates the stability of a cluster relative to its neighbours. As shown in table 2, this is found to be more positive for $\mathrm{Zn}_{3} \mathrm{~S}_{3}$ cluster. In $\mathrm{Zn}_{2} \mathrm{~S}_{2}$, although there are $\mathrm{Zn}-\mathrm{Zn}$ and $\mathrm{S}-\mathrm{S}$ interactions which increase its binding energy, it has nonlinear $\mathrm{S}-\mathrm{Zn}-\mathrm{S}$ bond angles and therefore, it is less stable than $\mathrm{Zn}_{3} \mathrm{~S}_{3}$ and $\mathrm{Zn}_{4} \mathrm{~S}_{4}$. Thus the negative value of $\Delta^{2} E\left(\mathrm{Zn}_{2} \mathrm{~S}_{2}\right)$ just indicates that it is less stable in comparison to $\mathrm{Zn}_{3} \mathrm{~S}_{3}$. In $\mathrm{Zn}_{4} \mathrm{~S}_{4}$, there is almost linear $\mathrm{S}-\mathrm{Zn}-\mathrm{S}$ bond angle but there is no $\mathrm{Zn}-\mathrm{Zn}$ and $\mathrm{S}-\mathrm{S}$ homo-nuclear interaction. $\mathrm{Zn}_{3} \mathrm{~S}_{3}$ has near-linear $\mathrm{S}-\mathrm{Zn}-\mathrm{S}$ bond angles and also has considerable $\mathrm{Zn}-\mathrm{Zn}$ interaction which makes it most stable. $\mathrm{Zn}_{6} \mathrm{~S}_{6}$ is a three-dimensional structure and stability is achieved via competition between existence of linear $\mathrm{S}-\mathrm{Zn}-\mathrm{S}$ bond angles and larger coordination numbers, in which the latter wins. This structure is the smallest cage-like structure and is a motif for the bulk wurtzite $\mathrm{ZnS}$ structure.

\section{Conclusions}

$\mathrm{Zn}_{n} \mathrm{~S}_{n}$ clusters are planar ring-like structures and are highly symmetric up to $n=4$. Cluster with $n=5$ is semi-planar while clusters with $n>5$, are cage-like structures. The binding energy increases with increase in the size of cluster, as expected. The difference between the vertical detachment energy and the electron affinity (VDE - AE) is related to $\eta$, the global hardness parameter, and is found to be highest for $\mathrm{Zn}_{3} \mathrm{~S}_{3}$. The second difference in energy that indicates the stability of a cluster with respect to its neighbours, is also highest for $\mathrm{Zn}_{3} \mathrm{~S}_{3}$ cluster, as shown in table 2. Slight $\mathrm{Zn}-\mathrm{Zn}$ and $\mathrm{S}-\mathrm{S}$ homo-polar interaction, seen in $\mathrm{Zn}_{3} \mathrm{~S}_{3}$ and not observed for other clusters, adds more strength to binding. From table 1, it may be seen that $\mathrm{Zn}_{3} \mathrm{~S}_{3}$ is a possible fragment in the least resistant fragmentation channel for clusters $\mathrm{Zn}_{5} \mathrm{~S}_{5}, \mathrm{Zn}_{6} \mathrm{~S}_{6}$ and $\mathrm{Zn}_{7} \mathrm{~S}_{7}$ and in the next possible fragmentation channel for clusters $\mathrm{Zn}_{8} \mathrm{~S}_{8}$ and $\mathrm{Zn}_{9} \mathrm{~S}_{9}$. It is, therefore, inferred that $\mathrm{Zn}_{3} \mathrm{~S}_{3}$ is the most stable and $\mathrm{Zn}_{7} \mathrm{~S}_{7}$ is the least stable cluster among the clusters studied in this work. $\mathrm{Zn}_{4} \mathrm{~S}_{4}$ cluster shows more semi-metallicity as compared to other clusters. All planar clusters possess more ionic character than covalent character. Weak $\mathrm{Zn}-\mathrm{Zn}$ homonuclear bonding is seen in $\mathrm{Zn}_{3} \mathrm{~S}_{3}$ cluster. $\mathrm{S}-\mathrm{S}$ interaction is seen only in $\mathrm{Zn}_{2} \mathrm{~S}_{2}$ and $\mathrm{Zn}_{3} \mathrm{~S}_{3}$ clusters. Clusters from $\mathrm{Zn}_{5} \mathrm{~S}_{5}$ to $\mathrm{Zn}_{9} \mathrm{~S}_{9}$ prefer to get fragmented mostly into $\mathrm{Zn}_{3} \mathrm{~S}_{3}$ units out of various fragmentation channels. This fact confirms that $\mathrm{Zn}_{3} \mathrm{~S}_{3}$ is the most stable cluster and serves as building unit for larger cage-like structures.

\section{Acknowledgements}

One of the authors (DLL) acknowledges the University Grants Commission, Government of India, for financial support and study leave under FIP-Xth plan. (AK) acknowledges financial support from the Department of Science and Technology and the Board of College and University Development, University of Pune, Pune. Authors wish to thank CDAC, Bangalore, for providing hpc facilities.

\section{References}

Becke A D 1993 J. Chem. Phys. 985648

Blöchl P E 1994 Phys. Rev. B50 17953

Burnin A and BelBruno J J 2002 Chem. Phys. Lett. 362341

Burnin A, Sanville E and BelBruno J J 2005 J. Phys. Chem. A109 5026

Chuchev K and BelBruno J J 2005 J. Phys. Chem. A109 1564

Hamad S and Catlow C R A 2005 J. Phys. Chem. B109 2703

Hohenberg P and Kohn W 1964 Phys. Rev. 136 B864

Katircioğlu S and Erkoc S 2001 J. Mol. Struct. (THEOCHEM) $\mathbf{5 4 6} 99$

Kohn W and Sham L J 1965 Phys. Rev. 140 A1133

Kresse G and Furthmüller J 1996a Comput. Mater. Sci. 615

Kresse G and Furthmüller J 1996b Phys. Rev. B59 1758

Kresse G and Joubert J 1999 Phys. Rev. B59 1758

Kresse G and Furthmüller J 2001 Vienna ab initio simulation package (Wien: Technische Universitat) (http://cms.mpi.univie. ac.at/vasp)

Kumbhojkar N, Nikesh V V, Mahamuni S and Kshirsagar A 2000 J. Appl. Phys. 886260

Lee C, Yang W and Parr R G 1988 Phys. Rev. B37 785

Maxtain J M, Fowler J E and Ugalde J M 2001 Phys. Rev. A61 053201

Ndukwe I C 1996 Solar Energ. Mater. Solar C. 40123

Niemegeers A and Burgelman M 1997 J. Appl. Phys. 812881

Perdew J, Burke K and Ernzerhof M 1996 Phys. Rev. Lett. 773865

Sebastian P J and Ocampo M 1996 Sol. Energ. Mater. Solar C. 441

Verma A K, Rauchfuss T B and Wilson S R 1995 Inorg. Chem. 34 3072 